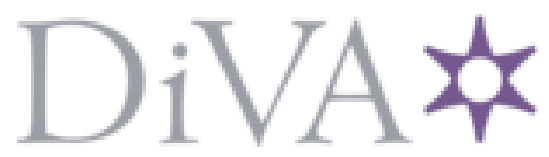

http://www.diva-portal.org

This is the published version of a paper published in Physical Review E. Statistical, Nonlinear, and Soft Matter Physics.

Citation for the original published paper (version of record):

Gonoskov, A., Korzhimanov, A., Kim, A., Marklund, M., Sergeev, A. (2011)

Ultrarelativistic nanoplasmonics as a route towards extreme-intensity attosecond pulses.

Physical Review E. Statistical, Nonlinear, and Soft Matter Physics, 84(4): 046403

http://dx.doi.org/10.1103/PhysRevE.84.046403

Access to the published version may require subscription.

N.B. When citing this work, cite the original published paper.

Permanent link to this version:

http://urn.kb.se/resolve?urn=urn:nbn:se:umu:diva-4996 1 


\title{
Ultrarelativistic nanoplasmonics as a route towards extreme-intensity attosecond pulses
}

\author{
A. A. Gonoskov, ${ }^{1,2}$ A. V. Korzhimanov, ${ }^{1,2}$ A. V. Kim, ${ }^{1}$ M. Marklund, ${ }^{2}$ and A. M. Sergeev ${ }^{1}$ \\ ${ }^{1}$ Institute of Applied Physics, Russian Academy of Sciences, 603950 Nizhny Novgorod, Russia \\ ${ }^{2}$ Department of Physics, Umea University, SE-901 87 Umeå, Sweden
}

(Received 22 September 2010; revised manuscript received 29 July 2011; published 10 October 2011)

\begin{abstract}
The generation of ultrastrong attosecond pulses through laser-plasma interactions offers the opportunity to surpass the intensity of any known laboratory radiation source, giving rise to new experimental possibilities, such as quantum electrodynamical tests and matter probing at extremely short scales. Here we demonstrate that a laser irradiated plasma surface can act as an efficient converter from the femto- to the attosecond range, giving a dramatic rise in pulse intensity. Although seemingly similar schemes have been described in the literature, the present setup differs significantly from the previous attempts. We present a model describing the nonlinear process of relativistic laser-plasma interaction. This model, which is applicable to a multitude of phenomena, is shown to be in excellent agreement with particle-in-cell simulations. The model makes it possible to determine a parameter region where the energy conversion from the femto- to the attosecond regime is maximal. Based on the study we propose a concept of laser pulse interaction with a target having a groove-shaped surface, which opens up the potential to exceed an intensity level of $10^{26} \mathrm{~W} / \mathrm{cm}^{2}$ and observe effects due to nonlinear quantum electrodynamics with upcoming laser sources.
\end{abstract}

DOI: 10.1103/PhysRevE.84.046403

PACS number(s): 52.38.-r, 42.65.Ky, 42.65.Re, 52.27.Ny

\section{INTRODUCTION}

Recent progress in ultrahigh-power laser technology has resulted in pulse intensities surpassing $10^{22} \mathrm{~W} / \mathrm{cm}^{2}[1]$ and stimulated the construction of multi-petawatt laser sources [2]. Such lasers open up opportunities for studying both a number of fundamentally new problems, such as the effects of vacuum nonlinearities [3-5] in laser fields and photonuclear physics, as well as some very important applications, for example, laser based particle acceleration, fast ignition fusion schemes, and the generation of electromagnetic radiation with tailored properties.

Given this, the study of overdense plasmas irradiated by relativistically intense laser pulses is a very important and challenging research trend. Numerical studies using the particlein-cell approach, with allowance for most important effects for the typical range of parameters, are known to be an excellent tool in this field, and the numerical results in general agree well with the experimental results. Furthermore, the so-called nonlinear fluid model [6] gives a set of equations analytically describing such processes, but the strongly nonlinear plasma behavior, due to the ultrarelativistic motion of the plasma electrons, makes the development of theoretical approaches a highly complicated task. Thus, one is normally forced to limit oneself to qualitative analyses and use a phenomenologically motivated ad hoc treatment.

The generation of high harmonics from intense laserplasma interactions is an intensely studied research field, with manifold applications [7], including the idea of reaching the extreme intensities needed to probe vacuum nonlinearities using lasers [8-12]. As of today, the most prominent theoretical model used in the analysis of such high-order harmonics generation (HHG) is the so-called oscillating mirror model (OMM). In the OMM, one considers the backradiation from an overdense plasma by taking into account the retarded emission from the oscillating source. This approach was first proposed by Bulanov et al. [13] and developed further in Refs. [14,15]. Lately, the OMM approach has been reexamined by Gordienko et al. [16], who proposed that at each moment of time there exists a so-called apparent reflection point (ARP) at which the energy flux vanishes. This assumption implies a local (in time) energy conservation or, phrased differently, the approach neglects the energy accumulated by the plasma, in the form of the fields due to charge separation caused by the light pressure. An asymptotic analysis of the ARP dynamics in the strongly relativistic limit [17] indicates the universal properties of the HHG spectra: The intensity of $n$th harmonic scales as $n^{-8 / 3}$, and the cutoff $\sim \gamma_{\max }^{3}$, where $\gamma_{\max }$ is the maximal relativistic factor of the ARP. These results agree with the experiment $[18,19]$.

Nevertheless, the assumption of the OMM concerning local temporal energy conservation is valid only for restricted values of the plasma density and laser intensity. The dimensionless parameter $\delta$ used in Fig. 1 is the energy dynamically accumulated by plasma, which is defined as

$$
\delta=\frac{E_{p}^{\max }-E_{p}^{\mathrm{fin}}}{E_{\text {cycle }}},
$$

where $E_{p}^{\max }$ and $E_{p}^{\mathrm{fin}}$ are the maximum and final values of the energy accumulated in the form of the plasma internal fields and electrons motion in the process of plasma interaction with one cycle of radiation with energy $E_{\text {cycle }}$; that is, parameter $\delta$ describes part of the energy accumulated by plasma that is later reemitted back. In the bottom left corner of the figure we find the zone labeled "OMM," for which $\delta \ll 1$. Here the energy accumulation can be neglected as assumed in the OMM. In the top right corner the region of relativistically self-induced transparency (RSIT) is shown. Thus, there is a large, and very important parameter region that so far has not been covered by any theoretical model and for which $\delta \sim 1$ such that the OMM's assumption of local energy conservation is no longer valid. Particle-in-cell (PIC) simulations indicate that here collective ultrarelativistic electron motion can give rise to nanoplasmonic structures (nanometer scale surface layers) and their oscillations, which provide at each period the 


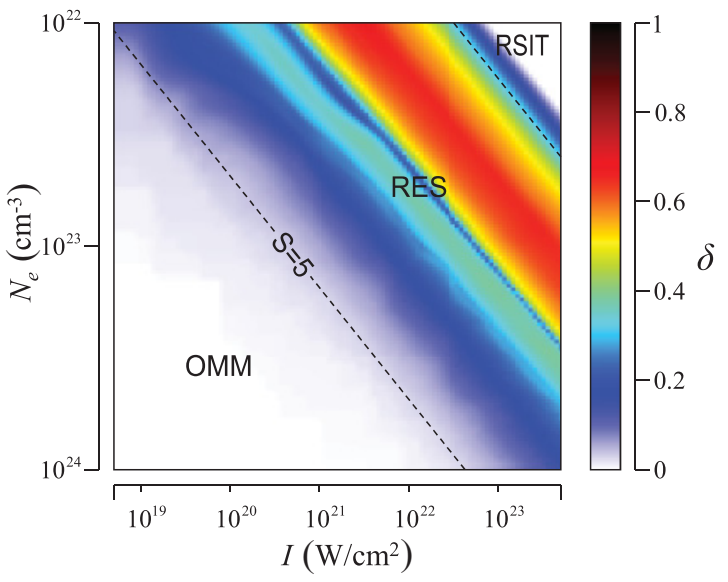

FIG. 1. (Color online) The dynamically accumulated by plasma energy $\delta$ obtained from one-dimensional PIC simulations of a plasma with density $N_{e}$ at oblique irradiation $\left(\theta=60^{\circ}, p\right.$ polarization) by a wave with constant intensity $I$ and $1 \mu \mathrm{m}$ wavelength during one optical cycle. $S$ is the ultrarelativistic similarity parameter, defined as the quotient between dimensionless density and the dimensionless amplitude [see Eq. (3)].

laser pulse energy conversion to the energy of internal electric and magnetic plasma fields and consequent reemission in the form of attosecond burst. The process results in a markedly slower decay in the generated higher harmonic spectra [20] as compared to the OMM results. Furthermore, based on the phenomenological assumption of electron nanobunches appearing in the plasma and emitting radiation, it was shown in Ref. [21] that the spectra can be much flatter than predicted by the OMM. Moreover, the OMM assumes as a prerequisite that the incident and backradiated amplitudes at the ARP are equal, in accordance with the Leontovich boundary conditions (which is in direct correspondence to the local energy conservation). Consequently, situations where large field amplifications are to be expected cannot be analyzed using this model. Thus, finding a new theoretical model in the relevant parameter regime between the OMM and RSIT regions is of utmost importance for a large number of applications.

In the present work we propose a physically motivated model, the so-called relativistic electronic spring (RES) model describing the highly nonlinear behavior of laser-plasma interactions. The model gives very good agreement with simulation and makes it possible to analytically study a vast range of regimes in laser-plasma interactions that otherwise would be out of reach for analysis. In particular, one of the most remarkable effects in the RES regime is the possibility of generating attosecond pulses with an amplitude several orders of magnitude higher than the incident laser pulse. Here we apply the RES model to this amplification effect in order to understand the underlying physical mechanisms and determine the optimal parameters for an experiment to be performed. We compare the results with particle-in-cell simulations and find excellent agreement. The implications of our results are discussed, in particular, the possibility to utilize this new type of secondary source for novel experiments.

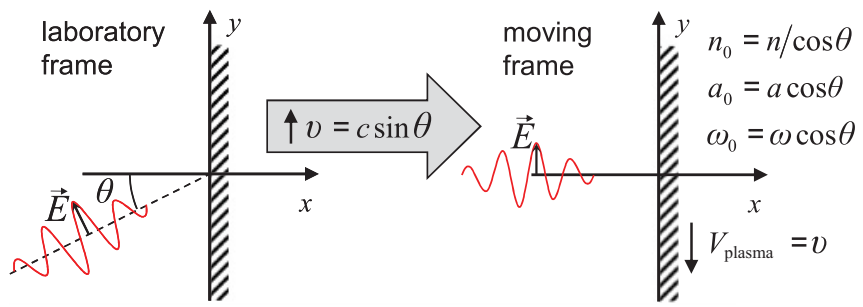

FIG. 2. (Color online) Transformation to a moving reference frame. Here the plasma density $n$, wave amplitude $a$, and frequency $\omega$ have subscript 0 denoting the moving frame.

\section{ULTRARELATIVISTIC ENERGY CONVERSION ON THE SURFACE OF A PLANAR TARGET}

The process of energy conversion due to the oblique incidence of a $p$-polarized electromagnetic wave on a plane plasma boundary $(x=0, y, z)$ may be considered using a boosted frame moving along the plasma surface and plane of incidence in the $y$ direction with the velocity $c \sin \theta$, where $c$ is the speed of light and $\theta$ is the angle of incidence (see Fig. 2), thus making the problem one-dimensional [22].

The incident laser pulse pushes the electrons into the plasma due to the light pressure. Unlike the case of normal incidence, the oblique incidence results in the emergence of uncompensated currents and magnetic fields in the boosted frame. Therefore, the electrons experience an additional ponderomotive action, which is different during the two half periods of the incident wave. When the laser electric field is directed along the $y$ axis, the Lorentz force due to uncompensated currents enhances the light pressure effect, pushing the electrons further from the boundary. At ultrarelativistic intensities the shifted electrons form a thin layer, where the charge and current densities greatly exceed the ones in the unperturbed plasma. At this stage, the incident wave energy is transformed to the kinetic energy of the particles and the energy of the internal plasma fields caused by charge and current separation.

The formation of an ultrathin (nanoscale) electron layer due to the interaction between an ultrarelativistic laser pulse and an overdense plasma has been known for about a decade, reported in the works on relativistic self-induced transparency [23,24] and particle acceleration using thin foils [25]. Unlike in the case of a circularly polarized laser pulse, in which electrons may be shifted by a distance of several wavelengths, a linearly $p$-polarized pulse results in a light pressure force that oscillates within a field period. Hence, the electrons are pushed from the surface for no longer than a fraction of the optical wavelength, then break away under the action of the charge separation force and travel toward the incident wave in the form of a thin current layer, providing a source of attosecond burst. The above described processes can clearly be seen in Fig. 3(a), where the results of 1D PIC simulation are presented.

Thus, the described process and concomitant energy conversion may be represented as a sequence of three stages: (1) pushing of electrons from the surface by the ponderomotive force and formation of a thin current layer giving an energy transfer from the laser field to the plasma fields and electrons; (2) backward accelerated motion of the current layer toward the incident wave with the conversion of the energy accumulated 

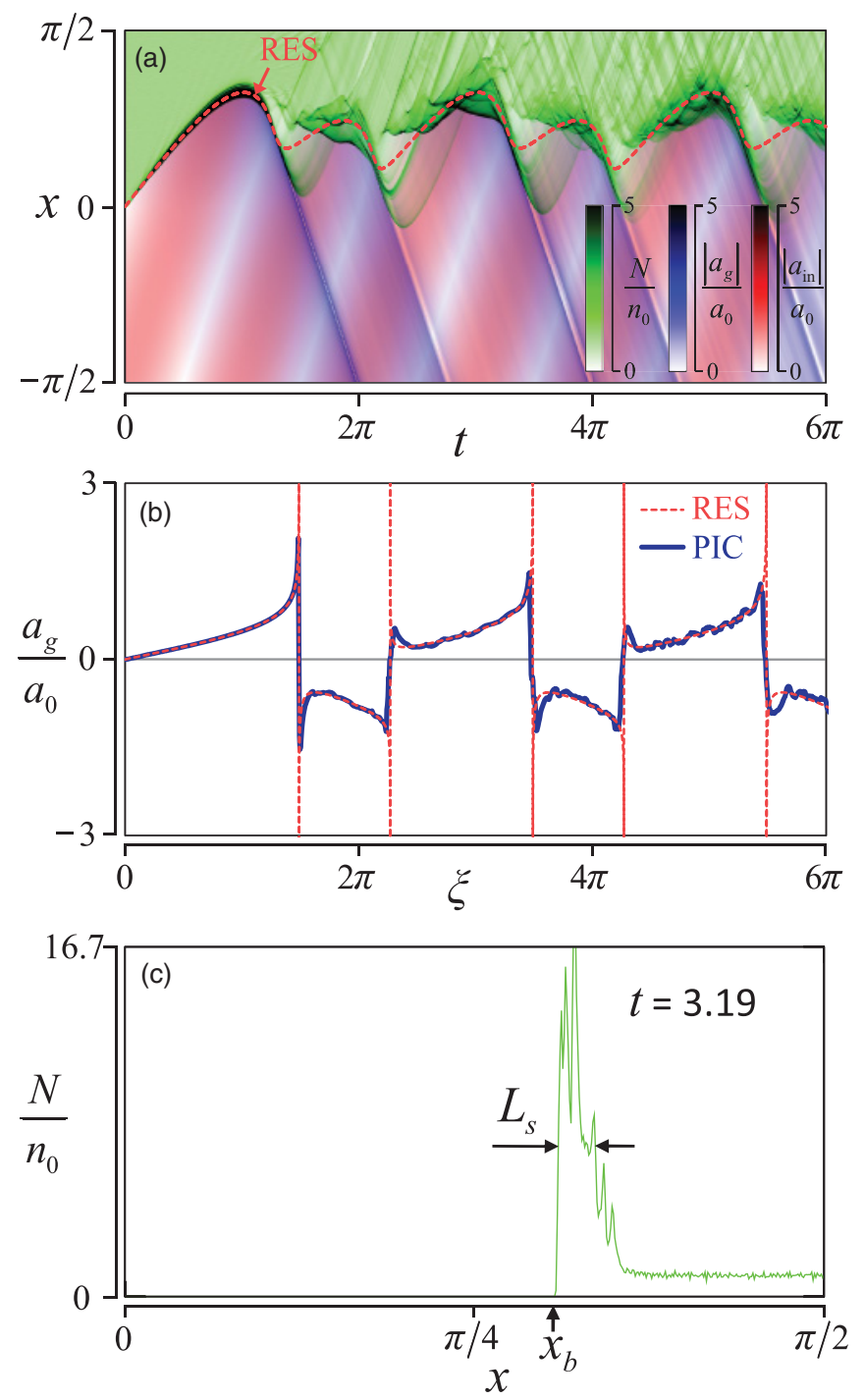

FIG. 3. (Color online) (a) Space-time distribution of electrons density $N$ (green) and amplitudes of incident $a_{\text {in }}$ (red) and backradiated $a_{g}$ (blue) electromagnetic fluxes obtained from 1D PIC simulation of plasma with density $4 \times 10^{23} \mathrm{~cm}^{-3}$ at oblique irradiation $\left(\theta=11.25^{\circ}\right)$ by a wave with constant intensity $5 \times 10^{22} \mathrm{~W} / \mathrm{cm}^{2}$ and $1 \mu \mathrm{m}$ wavelength during three optical cycles. The dashed red curve shows position of the thin layer obtained using the RES model. Time and coordinate are in dimensionless units [see Eq. (3)]. (b) Backradiated signal obtained by PIC simulation (blue curve) and using the RES model (dashed red curve). (c) Electron density distribution $N(x)$ at the instant of maximum displacement $(t=3.19)$.

in the plasma and laser field energy into the layer electrons kinetic energy; and (3) radiation of attosecond pulses by a formated ultrarelativistic electron bunch due to conversion of the kinetic energy and laser field energy to the XUV and x-ray range. Based on the motion of the plasma electrons and the energy conversion scenario we find that it is natural to refer to this three-step process as to a model of a RES. It should be emphasized that due to the energy accumulation in the plasma, the backradiated field can be much larger than the incident field. This is the fundamental difference from the OMM (see, e.g., [26,27]), a model which assumes the incident and backradiated fields equality at some oscillating point called ARP.

\section{THE RES MODEL}

The PIC simulations indicate that under the laser radiation pressure the electrons are shifted and group into a thin boundary layer. To understand the underlying physics of this phenomenon we made the following rough estimate for the boundary layer thickness in the quasistatic approximation at the instant of maximum displacement. The balance of the forces acting on an arbitrary electron with coordinate $x$ inside the layer near the boundary is given by

$$
\begin{aligned}
& n_{0} \frac{x}{\cos \theta}-\int_{x_{b}}^{x} N(\chi) \frac{d \chi}{\cos \theta} \\
& \quad=v_{y}(x)\left[2 a_{0}+n_{0} x \tan \theta-\int_{x_{b}}^{x} N(\chi) v_{y}(\chi) \frac{d \chi}{\cos \theta}\right],
\end{aligned}
$$

where the left-hand side corresponds to the electric part of the Lorentz force, whereas the terms on the right-hand side describe the magnetic part due to the incident radiation magnetic field and the magnetic field generated by the ion and electron currents, respectively; $a_{0}$ and $n_{0}$ are the radiation electric field amplitude and unperturbed plasma density in the moving frame, $x_{b}$ is the coordinate of the frontier electron between the plasma and vacuum [see Fig. 3(c)], $N(\chi)$ is the electron density distribution, and $v_{y}(\chi)$ is the distribution of the electrons velocity $y$ component (in the speed of light units), which is the same for all electrons with coordinate $\chi$ in the quasistatic approximation due to generalized transverse momentum conservation. The plasma ions assumed to be immobile in the laboratory frame. We use dimensionless quantities which can be expressed in terms of dimensional time $\hat{t}$, coordinate $\hat{x}$, density $\hat{n}$, and electric field amplitude $\hat{a}$ according to

$$
t=\omega \cos \theta \hat{t}, \quad x=\frac{\omega \cos \theta}{c} \hat{x}, \quad n=\frac{4 \pi e^{2}}{m \omega^{2}} \hat{n}, \quad a=\frac{e}{m c \omega} \hat{a},
$$

where $\omega$ is the carrier laser frequency, while $m$ and $e$ are the electron mass and charge, respectively.

The first-order consideration $\left(x=x_{b}\right)$ of Eq. (2) gives the layer position in the form

$$
x_{s}=\frac{2 a_{0} \cos \theta}{n_{0}(1-\sin \theta)},
$$

while the second-order consideration $\left(x=x_{b}+d x, d x \ll 1\right)$ in the ultrarelativistic limit $\left(v_{y} \approx 1\right)$ gives the estimate for the electron density near the boundary in the form

$$
n_{s} \approx n_{0} \frac{1-\sin \theta v_{y}\left(x_{s}\right)}{1-v_{y}^{2}\left(x_{s}\right)} \approx p_{y}^{2}\left(x_{s}\right)(1-\sin \theta),
$$

where $p_{y}(x)$ is the electron momentum $y$ component. Assuming that the layer has a squared shape for the electron density distribution and contains all the electrons shifted from the region $\left(0<x<x_{s}\right)$, we can estimate the layer thickness:

$$
L_{s} \approx \frac{2 a_{0} \cos \theta}{n_{0}(1-\sin \theta)^{2}} \frac{1}{p_{y}^{2}} .
$$


The vector potential $y$ component can be estimated through integrating from infinity to $x$ the $z$ component of the magnetic field of the current separation, neglecting the magnetic field of the laser radiation:

$$
A_{y}\left(x_{s}\right) \approx \frac{a_{0}}{\cos \theta(1-\sin \theta)} L_{s} .
$$

Finally, using (6), (7), and the generalized transverse momentum conservation $\left(p_{y}-A_{y}=\sin \theta\right)$ we derive the estimate for the layer thickness $L_{s}$ :

$$
L_{S} \propto S^{-\frac{1}{3}} a^{-\frac{2}{3}},
$$

where $S=a / n$ is the relativistic similarity parameter [28], while $a$ and $n$ are the dimensionless electric field amplitude and unperturbed plasma density in the laboratory frame, respectively. Despite the roughness of the involved assumptions, the negative power of the amplitude in the obtained estimate allows us to make an important conclusion: The thickness of the boundary layer tends to zero as the laser intensity increases and, in the ultrarelativistic limit, is much smaller than both the laser wavelength and the shift deepness $x_{s}=$ $2 S^{-1} \cos ^{3} \theta /(1-\sin \theta)$ [see Eq. (4)]; that is, it is negligible in comparison with all the other spatial scales involved in the process. This analytical consideration demonstrates that this phenomenon is not caused by the sharp plasma boundary, but is related to the ultrarelativistic character of the electrons motion.

A model describing the dynamics of the thin boundary layer and the generation of attosecond pulses may be formulated starting from three intuitively clear and physically justified prerequisities that can be verified using PIC simulation. First, we assume that at each moment of time the plasma electrons are represented by two fractions: one infinitely narrow layer of shifted electrons at a certain moving point $x_{s}$, where all the electrons from the region $0<x<x_{s}$ are accumulated, and one population of electrons with unperturbed density at $x>x_{s}$. As all the electrons within the boundary layer have the same velocity $x$ component $\beta_{x}$ moving the same way along the $x$ direction, in the ultrarelativistic limit these electrons have the same velocity $y$ component $\beta_{y}^{2} \approx 1-\beta_{x}^{2}$ as well, which is the second assumption. Third, we suppose that the motion of the electrons in the boundary layer together with the flow of uncompensated ions in the $0<x<x_{s}$ region generates the radiation which completely compensates the incident electromagnetic radiation in the unperturbed part of the plasma at $x>x_{s}$.

It is readily shown from Maxwell's equations that in a one-dimensional geometry a moving charged layer with surface charge $\sigma$ emits electromagnetic flows with amplitudes $2 \pi \sigma \beta_{y} /\left(1-\beta_{x}\right)$ and $2 \pi \sigma \beta_{y} /\left(1+\beta_{x}\right)$ in the positive and negative directions of the $x$ axis, respectively. Consequently, the expression for the incident wave compensation may be written in the form

$$
\sin \left(x_{s}-t\right)=\frac{S}{2 \cos ^{3} \theta}\left(\sin \theta-\frac{\beta_{y}}{1-\beta_{x}}\right) x_{s},
$$

where the left-hand side corresponds to the incident wave, whereas the terms on the right-hand side describe the radiation of the uncompensated ions and the boundary layer, respectively. Analogous to Eq. (9), the electric field, as a function of retarded time $\xi=x+t$, emitted by the plasma in the negative $x$ direction is given by

$$
a_{g}\left[\xi=x_{s}(t)+t\right]=a_{0} \frac{S}{2 \cos ^{3} \theta}\left(\frac{\beta_{y}}{1+\beta_{x}}-\sin \theta\right) x_{s}(t),
$$

where $a_{0}=a \cos \theta$ is the incident wave amplitude in the boosted frame.

The layer dynamics is determined by the equation

$$
\frac{d}{d t} x_{s}=\beta_{x}
$$

with the initial condition $x_{s}(t=0)=0$. By virtue of the ultrarelativistic motion, the position of the layer may be found by assuming that the full particle velocity is equal to the speed of light; that is, $\beta_{x}^{2}+\beta_{y}^{2}=1$. Equations (9) and (11) are then self-consistent and the layer motion is described by a first-order nonautonomous ordinary differential equation or by an autonomous system

$$
\begin{aligned}
\frac{d u}{d \tau} & =\frac{\left(u^{2}-1\right)(\sin \theta-u) \pm \frac{4 \cos ^{3} \theta}{S}\left[1-\eta^{2}(\sin \theta-u)^{2}\right]^{\frac{1}{2}}}{\eta\left(u^{2}+1\right)} \\
\frac{d \eta}{d \tau} & =\frac{u^{2}-1}{u^{2}+1}
\end{aligned}
$$
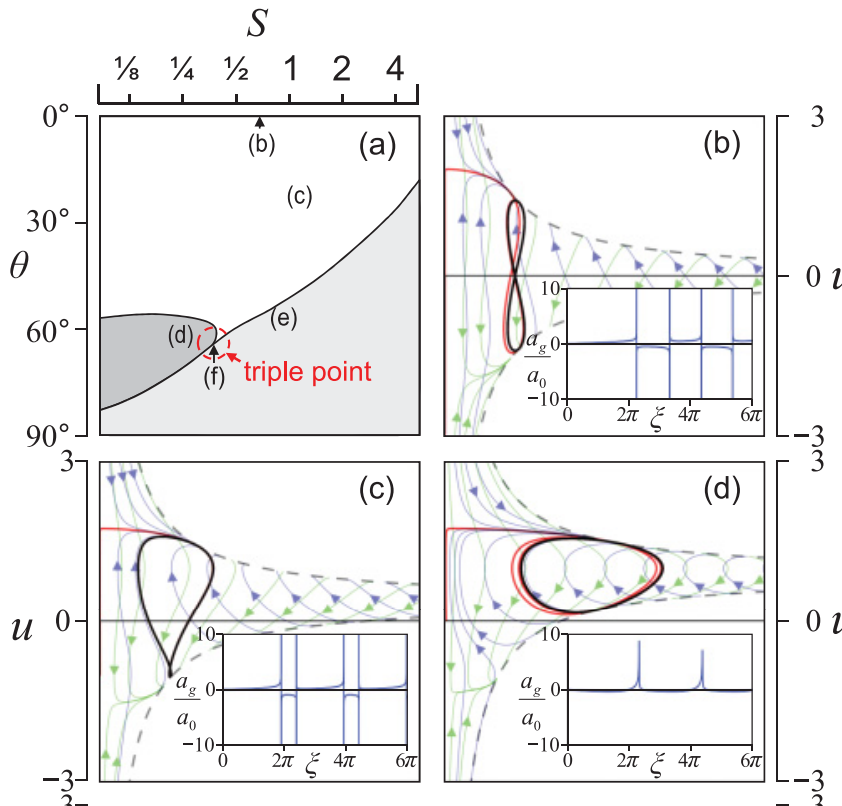

3

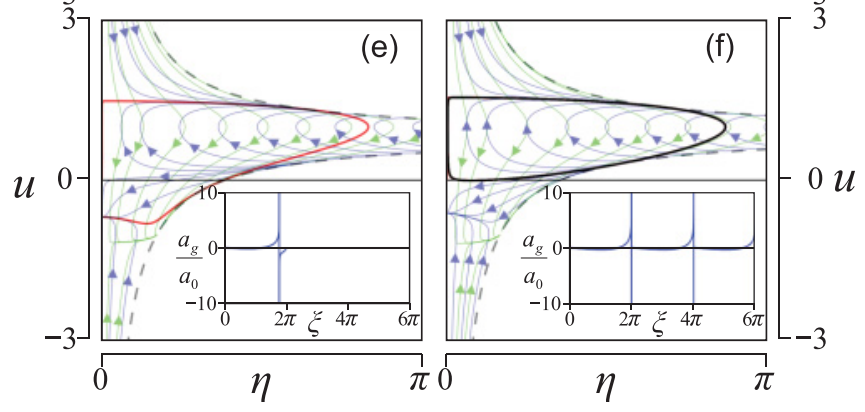

FIG. 4. (Color online) (a) Regions on the plane of parameters $S, \theta$ corresponding to qualitatively different forms of solution of Eq. (12). (b)-(f) Form of solution on the plane $\{\eta, u\}$ (red), its limit cycle (black), lines of phase portrait for the "+" sign (blue) and the "-" sign (green) in Eq. (12), and the corresponding form of electromagnetic backradiation from the plasma. 
for the variables $\eta(\tau)=x_{s} S /\left(2 \cos ^{3} \theta\right), u(\tau)=\beta_{y} /\left(1-\beta_{x}\right)$, where $\tau=t S /\left(2 \cos ^{3} \theta\right)$. The solution of Eq. (12) depends on two dimensionless variables $S$ and $\theta$ and may be analyzed in the plane $\{\eta, u\}$, where we have two sheets corresponding to the choice of sign in Eq. (12).

The topology of the phase plane is characterized by the existence of a stable limit cycle (see Fig. 4). It is convenient to classify the form of the solution by the number of $\eta$-axis intersections of $u(\tau)$ or, equivalently, by $\beta_{y}(t)$ sign changes in the $\eta>0\left(x_{s}>0\right)$ region. This takes place when $\beta_{x} \rightarrow-1$ and Eq. (10) becomes singular, which corresponds to the emission of the attosecond burst. There may occur either two such events [Fig. 4(c)], one [Fig. 4(e)], or none [Fig. 4(d)] in each optical period. Accordingly, two bipolar, one bipolar, or one unipolar attosecond pulses are generated. It is clear that we have the emission of two evenly spaced identical bursts in the case of normal incidence [Fig. 4(b)]. With increasing angle $\theta$, the second burst either disappears due to amplitude decay down to zero [(c) $\rightarrow$ (e) transition] or two bipolar pulses merge into one unipolar pulse as a result of convergence of their generation times [(c) $\rightarrow$ (d) bifurcation]. Our comprehensive numerical study indicates that in the ultrarelativistic case $I>$ $10^{21} \mathrm{~W} / \mathrm{cm}^{2}$ the results obtained using the RES model are in a very good agreement with the PIC simulations for all values of $\theta$ and for $S<5$ down to RSIT. As an example, a perfect quantitative agreement can be clearly seen in Figs. 3(a) and 3(b) (note that the agreement is obtained without using any adjustment parameters for the RES model). The RES model applicability region is labeled "RES" in Fig. 1.

Note that by a simple modification of Eqs. (9) and (10), the RES model can be easily generalized to take into account an arbitrary plasma density profile, as well as arbitrary laser pulse shape and polarization.

\section{GIANT ATTOSECOND PULSE GENERATION}

As can be seen in Fig. 3(b), the RES model describes the generation of attosecond pulses with the amplitude greatly exceeding the incident one, which we called a phenomenon of giant attosecond pulse generation. The phenomenon appears as a result of a coherent emission by the ultrarelativistic nanoplasmonic structure and is related to the singularity of the boundary layer radiation at the instant when $\beta_{y}$ changes the sign and $\beta_{x} \rightarrow-1$ [see Eq. (10)]. In order to find the amplitude and duration of the pulse generated near $\beta_{y}=0$ one has to take into account the finite value of the relativistic factor $\gamma=\left(1-\beta_{x}^{2}-\beta_{y}^{2}\right)^{-1 / 2}$, which is the external parameter for the RES model and can be taken, for example, from PIC simulation. Asymptotic analysis of the first term in the right-hand side of Eq. (10) near the point $\beta_{y}=0$ gives an analytical expression for the burst shape and its spectrum in the following form:

$$
a_{g}(\xi)=A_{g} f\left(\frac{\xi-\left.\xi\right|_{\beta_{y}=0}}{\tau_{g}}\right), \quad I_{k} \propto \exp \left(-\frac{k}{\alpha \gamma^{3}}\right)
$$

where $\alpha=\left.\frac{\partial \beta_{y}}{\partial t}\right|_{\beta_{y}=0}, f(v)=\frac{2 v}{v^{2}+1}, A_{g}=\frac{\left.a_{0} S \gamma x_{s}\right|_{y}=0}{2 \cos ^{3} \theta}$ is the pulse amplitude, $\tau_{g}=\left(2 \alpha \gamma^{3}\right)^{-1}$ is its characteristic duration, and $I_{k}$ is the intensity of the $k$ th harmonic. The value of $\alpha$ is assessed from the solution of the self-consistent system;

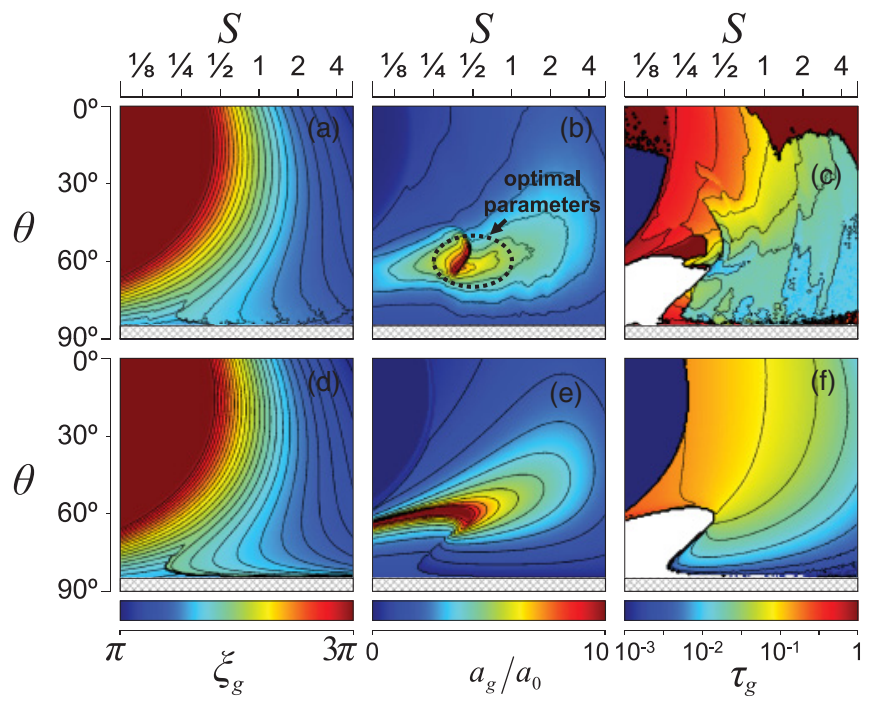

FIG. 5. (Color online) Giant pulse generation moment $\xi_{g}$, pulse amplitude $a_{g} / a_{0}$, and its duration $\tau_{g}$ obtained from the RES model with $\gamma=10$ and $\chi=56$ (d), (e), (f) and from 1D PIC simulations (a), (b), (c) of semi-infinite plasma obliquely irradiated by one optical cycle pulse with $1 \mu \mathrm{m}$ wavelength and amplitude $a=191.1$, which corresponds to $5 \times 10^{22} \mathrm{~W} / \mathrm{cm}^{2}$ intensity. For the PIC simulations, the pulse duration was assessed as a distance between maximum and minimum electric field points, and the region of unipolar pulse generation is given in white.

hence, it depends only on dimensionless parameters $S$ and $\theta$ and is independent of $\gamma$. This means that pulse duration in the ultrarelativistic limit tends to zero as $\gamma^{-3}$. Note that in the RES model the spectrum decays exponentially with the characteristic scale $\alpha \gamma^{3}$ and, in contrast to the OMM, has no region with a power-law decay. Thus, the RES region in Fig. 1 corresponds to a slower energy decay of the harmonics than the OMM region.

Depending on the thickness $l_{s}$ of the radiating electron layer, the radiation can be either coherent, for $l_{s}<\tau_{g}$, or incoherent, for $l_{s}>\tau_{g}$. In the latter case, when assessing the giant pulse amplitude one needs to take into consideration that only some fraction of electrons radiate coherently. The radiating electron layer thickness $l_{s}$ may be estimated assuming that, starting from the time of maximum displacement, it decreases proportionally to the number of electrons in the layer. Using the estimate for the layer thickness at the instant of maximum displacement (8) we can write the following estimate for the correction factor:

$$
C=\chi \frac{\tau_{g}}{l_{s}}=\chi \frac{1}{\alpha \gamma^{3}} \frac{x_{\max }}{\left.x_{s}\right|_{\beta_{y}=0}} S^{1 / 3} a^{2 / 3},
$$

where $x_{\max }$ is the maximum value of $x_{s}$ and $\chi$ is a dimensionless constant that is needed to account for arbitrary choice of the estimated values entering this expression.

In Fig. 5 the RES model results, that is, the results of the numerical solution of Eqs. (9) and (11), are compared with PIC simulations for different values of incidence angle $\theta$ and similarity parameter $S$. For all PIC simulations the intensity $I=5 \times 10^{22} \mathrm{~W} / \mathrm{cm}^{2}$, while the plasma density is determined in accordance with the value of $S$. The considered value of 
intensity also defines the parameter $\gamma$ for the RES model; so as can be seen from Figs. 5(c) and 5(f) the value $\gamma=10$ provides a fairly good quantative agreement for the giant pulse duration $\tau_{g}$, while an excellent agreement of the moment of giant pulse generation $\xi_{g}$ [see Figs. 5(a) and 5(d)] is not related to the choice of $\gamma$, because $\xi_{g}$ reaches asymptotics and changes insignificantly with the growth of $\gamma$ in the ultrarelativistic limit $\gamma \gtrsim 10$. The giant pulse amplitude determined with the RES model, including the correction factor (14) with $\chi=56$, is also in a good agreement with the PIC simulations results [see Figs. 5(b) and 5(e)].

The diagrams in Figs. 5(b) and 5(e) allow us to distinguish the zone with the center

$$
\theta_{g} \approx 62^{\circ}, \quad S_{g} \approx 1 / 2,
$$

and the boundaries $50^{\circ}<\theta<70^{\circ}, 1 / 4<S<1$ as the region of the most powerful attosecond pulse generation. The optimal parameters (15) correspond to the triple point in the $S$ and $\theta$ plane [Fig. 4(a)], which can be explained as follows. At the triple point [Fig. 4(f)], the limit cycle touches the $\eta$ axis, providing the longest time of electron moving with $\beta_{y} \approx 0$ and thus the largest duration of attosecond pulse generated by each electron. Therefore, this point corresponds to the optimal conditions for coherent radiation of all electrons within the boundary layer and consequently the maximum amplitude of the giant attosecond pulse, as coherency plays the dominant role.

Thus, both the RES model and the PIC simulations indicate the existence of optimal condition (15) for the phenomenon of giant attosecond pulse generation, which may serve as a guiding message for the basic experimental implementation: The phenomenon of giant attosecond pulse generation and concomitant anomalous efficient HHG can be observed in case of planar target oblique irradiation with incident angle $\theta \approx 62^{\circ}$ and dimensionless laser pulse amplitude $a$ and plasma density $n$ consistent, so that $n / a \approx 1 / 2$. The laser pulse contrast should, of course, be high enough but it is not a crucial point, as the steplike plasma density profile does not play the key role. To check the possibility of observing the phenomenon for lower intensities in Fig. 6 we plotted the amplitude increase factor $a_{g} / a_{0}$ as a function of plasma density $N_{e}$ and intensity

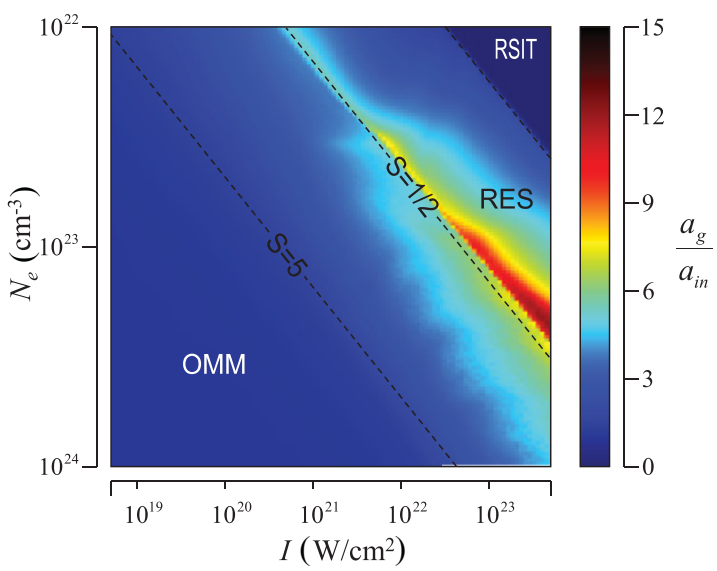

FIG. 6. (Color online) The amplitude increase factor $a_{g} / a_{0}$ obtained from 1D PIC simulations with the same parameters as in Fig. 1.
$I$ for the close to optimal incidence angle $\theta=60^{\circ}$. It is clearly seen that as a confirmation of the RES theory, the optimal conditions for the phenomenon are determined by the similarity parameter $S \approx 1 / 2$, while the amplitude increase factor depends on incident intensity and has a notable value $a_{g} / a_{0}>7$ at intensities $I>5 \times 10^{21} \mathrm{~W} / \mathrm{cm}^{2}$.

\section{SPACE FOCUSING FOR ATTOSECOND PULSES: A CONCEPT OF A GROOVE-SHAPED TARGET}

Based on the results obtained we propose a concept of extremely intense light generation at the level required for observation of the QED effects. The existence of the optimal incidence angle changes the presently accepted view of the spherical geometry as an optimal one for the attosecond radiation focusing mechanism. Our idea is to focus the giant burst formed in the regime described above by using a slightly grooved surface of the obliquely irradiated target at the optimal parameters (15), with the guiding line of the groove located in the plane of incidence [see Fig. 7(a)]. The PIC simulation of the proposed concept shows that the intensity $2 \times 10^{26} \mathrm{~W} / \mathrm{cm}^{2}$ can be reached in the zone with the size of order $10 \mathrm{~nm}$ with a 10-PW laser pulse, as can be seen in Fig. 7(b). In the laboratory frame the high field zone moves along the guiding line with speed $c / \sin \theta$. We note that the size of laser pulse along the transverse direction may be only a few wavelengths. The data shown in Fig. 5 may be used to modify surface profile and target density to allow using a laser pulse with a more complicated intensity profile in the transverse direction. Several PIC simulations of the proposed scheme with the parameter slightly varied close to the optimal ones (15) show that the effect is quite robust; thus, we consider the proposed concept to be very promising for

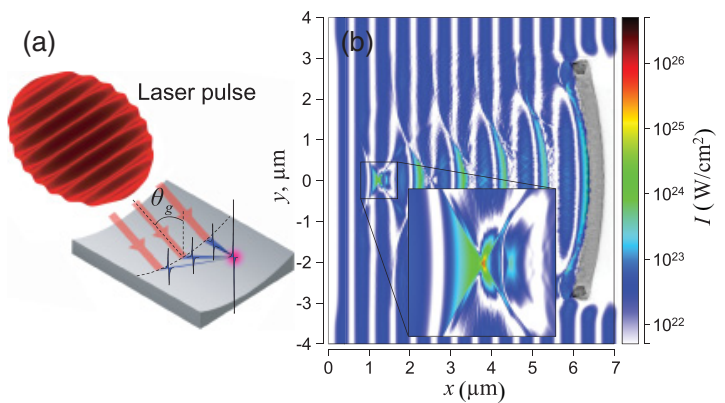

FIG. 7. (Color online) (a) Schematic representation of the concept of groove-shaped target. (b) Intensity distribution at focusing instant obtained from 2D PIC simulation: a linearly polarized wave with intensity $5 \times 10^{22} \mathrm{~W} / \mathrm{cm}^{2}$ and wavelength $1 \mu \mathrm{m}$ obliquely incident at an optimal angle $\theta_{g}=62^{\circ}$ on a parabolic groove-shaped target with density $0.85 \times 10^{23} \mathrm{~cm}^{-3}$, which corresponds to $S=0.4$. PIC simulation in the moving frame implies that the transverse size of the laser pulse is fairly large compared to the wavelength, which is a rather weak restriction. Fully relativistic parallel fast Fourier transform (FFT) based PIC code ELMIS [29] is used; an $8 \times 8-\mu \mathrm{m}$ region is represented by $8192 \times 8192$ cells; plasma ions are taken to be $\mathrm{Au}^{6+}$; each target cell contains approximately 100 virtual particles of each type; the time step is 3 as; the laser pulse front has a sine-squared profile with two wave periods duration. 
the experimental implementation in comparison with all the previously proposed concepts $[8,10]$.

\section{CONCLUSION}

In this work we studied the giant pulse generation process at oblique irradiation in an overdense plasma by a relativistically strong laser pulse. We analyzed the physics of ultrarelativistic nanoplasmonic structure formation and coherent emission as the origin of the giant attosecond pulse generation phenomenon. The model of RES was developed, providing a qualitative and, for some characteristics, also a fairly good quantitative description. The parameters of the most powerful burst generation (15) were determined. A concept of a groove- shaped target for high electromagnetic field generation aimed at obtaining the QED effects by means of upcoming laser sources was proposed and confirmed by PIC simulation.

\section{ACKNOWLEDGMENTS}

This research was supported by the Presidium of RAS, the RFBR (Grant No. 09-02-12322-ofi_m), the Presidential Council on Grants of the Russian Federation (Grant No. 3800.2010.2), the European Research Council (Grant No. 204059-QPQV), and the Swedish Research Council (Grant No. 2007-4422). We acknowledge the Joint Supercomputer Center of RAS and the Swedish National Infrastructure for Computing (SNIC) for the provided supercomputer sources.
[1] V. Yanovsky et al., Opt. Express 16, 2109 (2008).

[2] [http://www.extreme-light-infrastructure.eu].

[3] A. R. Bell and J. G. Kirk, Phys. Rev. Lett. 101, 200403 (2008).

[4] G. V. Dunne, H. Gies, and R. Schützhold, Phys. Rev. D 80, 111301 (2009).

[5] A. M. Fedotov, N. B. Narozhny, G. Mourou, and G. Korn, Phys. Rev. Lett. 105, 080402 (2010).

[6] A. I. Akhiezer and R. V. Polovin, JETP 3, 696 (1956).

[7] U. Teubner and P. Gibbon, Rev. Mod. Phys. 81, 445 (2009).

[8] S. V. Bulanov, T. Zh. Esirkepov, and T. Tajima, Phys. Rev. Lett. 91, 085001 (2003).

[9] N. M. Naumova et al., Phys. Rev. Lett. 92, 063902 (2004).

[10] S. Gordienko, A. Pukhov, O. Shorokhov, and T. Baeva, Phys. Rev. Lett. 94, 103903 (2005).

[11] F. Quéré et al., Phys. Rev. Lett. 96, 125004 (2006).

[12] A. Tarasevitch, K. Lobov, C. Wünsche, and D. von der Linde, Phys. Rev. Lett. 98, 103902 (2007).

[13] S. V. Bulanov, N. M. Naumova, and F. Pegoraro, Phys. Plasmas 1, 745 (1994).

[14] R. Lichters, J. M. ter Vehn, and A. Pukhov, Phys. Plasmas 3, 3425 (1996).

[15] D. von der Linde and K. Rzàzewski, Appl. Phys. B: Laser Opt. 63, 499 (1996).
[16] S. Gordienko, A. Pukhov, O. Shorokhov, and T. Baeva, Phys. Rev. Lett. 93, 115002 (2004).

[17] T. Baeva, S. Gordienko, and A. Pukhov, Phys. Rev. E 74, 046404 (2006).

[18] B. Dromey et al., Nat. Phys. 2, 456 (2006).

[19] B. Dromey et al., Nat. Phys. 5, 146 (2009).

[20] T. J. M. Boyd and R. Ondarza-Rovira, Phys. Rev. Lett. 101, 125004 (2008).

[21] D. an der Brügge and A. Pukhov, Phys. Plasmas 17, 033110 (2010)

[22] A. Bourdier, Phys. Fluids 26, 1804 (1983).

[23] F. Cattani, A. Kim, D. Anderson, and M. Lisak, Phys. Rev. E 62, 1234 (2000).

[24] V. I. Eremin, A. V. Korzhimanov, and A. V. Kim, Phys. Plasmas 17, 043102 (2010).

[25] A. A. Gonoskov, A. V. Korzhimanov, V. I. Eremin, A. V. Kim, and A. M. Sergeev, Phys. Rev. Lett. 102, 184801 (2009).

[26] G. A. Mourou, T. Tajima, and S. V. Bulanov, Rev. Mod. Phys. 78, 309 (2006).

[27] M. Marklund and P. K. Shukla, Rev. Mod. Phys. 78, 591 (2006).

[28] S. Gordienko and A. Pukhov, Phys. Plasmas 12, 043109 (2005).

[29] [http://www.ipfran.ru/english/structure/lab334/simlight.html]. 